\title{
On an Accelerated Overrelaxation Iterative Method for Linear Systems With Strictly Diagonally Dominant Matrix
}

\author{
By M. Madalena Martins*
}

\begin{abstract}
We consider a linear system $A x=b$ of $n$ simultaneous equations, where $A$ is a strictly diagonally dominant matrix. We get bounds for the spectral radius of the matrix $L_{r, \omega}$, which is accociated with the Accelerated Overrelaxation iterative method (AOR).
\end{abstract}

Sufficient conditions for the convergence of that method will be given, which improve the results of Theorem 3, Section 4 of [2], applied to this type of matrices.

1. Introduction. We want the solution $x$ of a linear system

$$
A x=b,
$$

where $A$ is an $(n, n)$ real-matrix and $x$ and $b$ are $n$-real-vectors. We assume $A$ is strictly diagonally dominant. There are several important iterative methods for the approximation of (1.1). We will take the (AOR) of [1]. For that, the matrix is expressed as the matrix sum

$$
A=I-E-F,
$$

where $I$ is the identity and $E$ and $F$ are respectively strictly lower and upper triangular $(n, n)$ matrices.

From the (AOR) method we can write the following equations:

$$
\begin{aligned}
x^{(i+1)}=(I-r E)^{-1}[(1-\omega) I+(\omega-r) E+\omega F] x^{(i)}+ & \omega(I-r E)^{-1} b, \\
& i=0,1,2, \ldots .
\end{aligned}
$$

So, $L_{r, \omega}$ will be the point-(AOR)-matrix associated with the matrix $A$,

$$
L_{r, \omega}=(I-r E)^{-1}[(1-\omega) I+(\omega-r) E+\omega F],
$$

and $\rho\left(L_{r, \omega}\right)$ the corresponding spectral radius.

2. Bounds for $\rho\left(L_{r, \omega}\right)$. As we assume $A$ strictly diagonally dominant, $A$ is nonsingular and verifies

$$
\left|a_{i i}\right|>\sum_{j=1 ; i \neq j}^{n}\left|a_{i j}\right|, \quad i=1,2, \ldots, n .
$$

Received October 18, 1979; revised March 18, 1980.

1980 Mathematics Subject Classification. Primary 65F10.

*This work was done when the author was "equiparado a bolseiro pelo Instituto Nacional de Investigação Científica". 
Bounds for $\rho\left(L_{r, \omega}\right)$ are obtained from

THEOREM 1. If $A$ of (1.1) is a strictly diagonally dominant matrix, then $\rho\left(L_{r, \omega}\right)$ satisfies the following:

$$
\begin{array}{r}
\min _{i} \frac{|1-\omega|-|\omega-r| e_{i}-|\omega| f_{i}}{1+|r| e_{i}} \leqslant \rho\left(L_{r, \omega}\right) \leqslant \max _{i} \frac{|\omega-r| e_{i}+|\omega| f_{i}+|1-\omega|}{1-|r| e_{i}} \\
i=1,2, \ldots, n
\end{array}
$$

where $|r|<1 / e_{i}$ and $e_{i}, f_{i}$ are respectively the i-row sums of the moduli of the entries of $E$ and $F$, respectively.

Proof. Since the eigenvalues of $L_{r, \omega}$ are given from

$$
\operatorname{det}\left(L_{r, \omega}-\lambda\right)=0
$$

after some manipulations, it is easy to verify that to solve (2.3) is equivalent to solving

$$
\operatorname{det} Q=0 \text {, }
$$

where $Q$ is

$$
Q=I-\frac{r(\lambda-1)+\omega}{\lambda-1+\omega} E-\frac{\omega}{\lambda-1+\omega} F
$$

If we take the parameter $r, \omega, \lambda$, in order that $Q$ be strictly diagonally dominant, we get

$$
|\lambda-1+\omega|>|\omega+(\lambda-1) r| e_{i}+|\omega| f_{i}, \quad i=1, \ldots, n .
$$

Then, the values of $\lambda$ satisfying (2.5) will not satisfy (2.4) and cannot be eigenvalues of (2.3). From (2.5), as $\lambda$ can take any value, we have

$$
|\lambda|-|1-\omega|>|\omega-r| e_{i}+|\lambda r| e_{i}+|\omega| f_{i}, \quad i=1, \ldots, n,
$$

or

$$
|\lambda|\left(1-|r| e_{i}\right)>|\omega-r| e_{i}+|\omega| f_{i}+|1-\omega|, \quad i=1, \ldots, n .
$$

Assuming $|r|<1 / e_{i}$, we have

$$
|\lambda|>\max _{i} \frac{|\omega-r| e_{i}+|\omega| f_{i}+|1-\omega|}{1-|r| e_{i}} .
$$

If $\lambda$ satisfies this inequality, it cannot be an eigenvalue of $L_{r, \omega}$, and then

$$
\rho\left(L_{r, \omega}\right) \leqslant \max _{i} \frac{|\omega-r| e_{i}+|\omega| f_{i}+|1-\omega|}{1-|r| e_{i}} \text { for }|r|<\frac{1}{e_{i}} .
$$

In order to get the lower bound, from (2.5) we write

$$
|1-\omega|-|\lambda|>|\omega-r| e_{i}+|\lambda r| e_{i}+|\omega| f_{i}, \quad i=1,2, \ldots, n,
$$

and 


$$
|\lambda|<\min _{i} \frac{|1-\omega|-|\omega-r| e_{i}-|\omega| f_{i}}{1+|r| e_{i}} .
$$

Since the values of $\lambda$ satisfying this inequality are not eigenvalues of $L_{r, \omega}$, then

$$
\rho\left(L_{r, \omega}\right) \geqslant \min _{i} \frac{|1-\omega|-|\omega-r| e_{i}-|\omega| f_{i}}{1+|r| e_{i}}, \quad i=1, \ldots, n \text {. Q.E.D. }
$$

As it is well known, for convenient values of $r$ and $\omega$, the (AOR) method becomes the well-known iterative methods:

$$
\begin{aligned}
r & =0, \omega=1 & & \text { Jacobi Method, } \\
r & =1, \omega=1 & & \text { Gauss-Seidel Method, } \\
r & =0, \omega & & \text { Simultaneous Overrelaxation Method, } \\
r & =\omega & & \text { Successive Overrelaxation Method. }
\end{aligned}
$$

Taking these values, we get from (2.2) the known results

$$
\begin{aligned}
& \rho\left(L_{0,1}\right) \leqslant \max _{i}\left(e_{i}+f_{i}\right), \\
& \rho\left(L_{1,1}\right) \leqslant \max _{i} \frac{f_{i}}{1-e_{i}}, \\
& \rho\left(L_{0, \omega}\right) \leqslant \max _{i}|\omega|\left(e_{i}+f_{i}\right)+|1-\omega|, \\
& \rho\left(L_{\omega, \omega}\right) \leqslant \max _{i} \frac{|\omega| f_{i}+|1-\omega|}{1-|\omega| e_{i}} .
\end{aligned}
$$

3. Convergence of the (AOR) Method.

THEOREM 2. If $A$ of (1.1) is a strictly diagonally dominant matrix and $\omega \geqslant r \geqslant 0$, then a sufficient condition for the convergence of the (AOR) method is

$$
0<\omega<\frac{2}{1+\max _{i}\left(e_{i}+f_{i}\right)} .
$$

Proof. From [1, Section 3], with $A$ strictly diagonally dominant, the (AOR) method is convergent if $0<\omega \leqslant 1,0 \leqslant r \leqslant 1$. From (2.2) we see that $\rho\left(L_{r, \omega}\right)$ will be less than 1 if

$$
|\omega-r| e_{i}+|\omega| f_{i}+|1-\omega|+|r| e_{i}<1, \quad i=1, \ldots, n \text {. }
$$

With $\omega>r \geqslant 0$ these conditions will be fulfilled.

Taking $f(\delta)=(\delta-r) e_{i}+\delta f_{i}+(1-\delta)+r e_{i}$, we see that $f(\delta)$ is a nonincreasing function of $\delta$ if $0 \leqslant \delta \leqslant 1$ and $f(0)=1, f(1)=e_{i}+f_{i}<1$. With $\delta>1, f(\delta)$ is an increasing function with $f(\delta)=1$ for $\delta=2 /\left(1+e_{i}+f_{i}\right)$. Then $\rho\left(L_{r . \omega}\right)<1$ if

$$
0<\omega<\frac{2}{1+\max _{i}\left(e_{i}+f_{i}\right)},
$$

and the (AOR) method will be convergent. Q.E.D. 
Let us consider a first-degree linear stationary iterative method

$$
x^{(i+1)}=G x^{(i)}+\left.d\right|_{i=0,1,2, \ldots},
$$

with $G$ a known $n \times n$ matrix, $d$ a known $n$-vector, and $x^{(0)}$ an arbitrary initial approximation for the solution $x$.

The following method

$$
x^{(i+1)}=[(1-\omega) I+\omega G] x^{(i)}+\left.\omega d\right|_{i=0,1,2, \ldots}
$$

will be called the extrapolated method of (3.2).

We recall now, the Theorem of Extrapolation [2, p. 2], as we need it in the sequel.

THEOREM 3 (THEOREM OF ExTRAPOLATION). The sufficient conditions for the convergence of (3.3) are:

(1) The original (3.2) is convergent,

(2) $0<\omega<2 /(1+\rho(G))$.

Setting $r=0$ in (1.3), we obtain

$$
x^{(i+1)}=[(1-\omega) I+\omega(E+F)] x^{(i)}+\left.\omega b\right|_{i=0,1,2, \ldots} .
$$

This is the extrapolated Jacobi method, where $\omega$ is the extrapolation parameter. After some computation, it is easy to verify that (1.3) is the extrapolated SOR method, when $r \neq 0$ and its extrapolation parameter is $\omega / r$.

THEOREM 4. If $A$ from (1.1) is strictly diagonally dominant, then $\rho\left(L_{0, \omega}\right)<1$ provided $0<\omega<2 /\left(1+\rho\left(L_{0,1}\right)\right)$.

Proof. Bearing in mind that $A$ strictly diagonally dominant implies $\rho\left(L_{0,1}\right)<1$ $[4$, p. 73], it is an immediate consequence of Theorem 3.

THEOREM 5. If $A$ of $(1.1)$ is strictly diagonally dominant, then $\rho\left(L_{r}, \omega\right)<1$ with $0<r \leqslant 1$ provided $0<\omega<2 r /\left(1+\rho\left(L_{r, r}\right)\right)$.

Proof. From (2.6d) we easily deduce that the SOR method will converge with $0<r \leqslant 1$ ( $A$ is strictly diagonally dominant).

Then, by the Theorem of Extrapolation, the AOR method will converge for $0<r \leqslant 1$ and $0<\omega<2 r /\left(1+\rho\left(L_{r, r}\right)\right)$.

THEOREM 6. The AOR method is convergent, i.e. $\rho\left(L_{r, \omega}\right)<1$, for:

(i) $0 \leqslant r \leqslant \omega$ and $0<\omega<2 /\left(1+\max _{i}\left(e_{i}+f_{i}\right)\right)$ if

$$
\frac{2 r}{1+\rho\left(L_{r, r}\right)} \leqslant \frac{2}{1+\max _{i}\left(e_{i}+f_{i}\right)}
$$

(ii) $0<r \leqslant 1$ and $0<\omega<2 r /\left(1+\rho\left(L_{r, r}\right)\right)$ or $1<r<\omega$ and $0<\omega<$ $2 /\left(1+\max _{i}\left(e_{i}+f_{i}\right)\right)$ if

$$
\frac{2 r}{1+\rho\left(L_{r, r}\right)}>\frac{2}{1+\max _{i}\left(e_{i}+f_{i}\right)} .
$$


Proof. These results come from Theorems 2 and 5. They improve the results of Theorem 3, Section 4 of [2], when the matrix $A$ of (1.1) is strictly diagonally dominant:

$$
\rho\left(L_{r, \omega}\right)<1 \quad \text { if } 0 \leqslant r<1 \text { and } 0<\omega \leqslant \max \left\{1, \frac{2 r}{1+\rho\left(L_{r, r}\right)}\right\} .
$$

Acknowledgment. The author wishes to thank Professor Fernanda de Oliveira and Professor Graciano de Oliveira for their suggestions and criticism during the writing of this paper.

Department of Mathematics

University of Coimbra

Coimbra, Portugal

1. A. HADJIDIMOS, "Accelerated overrelaxation method," Math. Comp., v. 32, 1978, pp. $149-157$.

2. A. HADJIDIMOS \& A. YEYIOS, The Principle of Extrapolation in Connection with the Accelerated Overrelaxation (AOR) Method, T. R. No. 16, Department of Mathematics, University of Ioannina, Ioannina, Greece, 1978.

3. G. AVDELAS, A. HADJIDIMOS \& A. YEYIOS, Some Theoretical and Computational Results Concerning the Accelerated Overrelaxation (AOR) Method, T. R. No. 8, Department of Mathematics, University of Ioannina, Ioannina, Greece, 1978.

4. R. S. VARGA, Matrix Iterative Analysis, Prentice-Hall, Englewood Cliffs, N. J., 1962.

5. D. M. YOUNG, Iterative Solution of Large Linear Systems, Academic Press, New York and London, 1971. 